\title{
RKKY-Reminiscent Interaction in Net Fractals
}

\author{
R. Jaroszewicz \\ Institute of Physics, Jan Długosz University \\ al. Armii Krajowej 13/15, 42-200 Czȩstochowa, Poland
}

\begin{abstract}
We define a specific class of fractals as "net fractals" and prove that in the logarithmic scale they are isomorphic with some bulk crystals. Furthermore, with the use of logarithmic coordinates, we prove that in the "net fractal" magnetic system the indirect exchange, by itinerant electrons can be presented in the form that is reminiscent of the Ruderman-Kittel-Kasuya-Yosida interaction characteristic of a system of fractional spectral dimension.
\end{abstract}

PACS numbers: $61.43 . \mathrm{Hv}, 75.30 . \mathrm{Hx}$

\section{Introduction}

Indirect magnetic interaction between spins arises due to the scattering of the electrons on the magnetic moments of the impurity ions. The conventional RudermanKittel-Kasuya-Yosida (RKKY) model of indirect coupling is valid provided that electron density is uniform and the dopant ions are distributed randomly within a matrix. However, in many cases the dopant ions show tendency towards clustering. These spontaneously patterned structures can be assembled in various geometries. The resulting clusters immersed within the matrix often show fractal symmetry. That is why different concepts which account for the mutual interplay of underlying topology and magnetic interactions are still under debate [1-5]. Evidently such a relation can be studied numerically, however, numerical calculations do not provide a simple understanding of the parameters that control the process. That is why even simplified analytical models are still attractive. We limit our study to "net fractals", a class of fractals, for which we show that after transition to logarithmic coordinates, the translation symmetry in the mass distribution is restored. It opens a possibility to describe the symmetries of some magnetic self-similar fractals in the way that is reminiscent of conventional RKKY formalism developed for crystalline systems.

\section{Model}

We say that $K \in R^{3}$ satisfies the scaling law $S$ or is infinite-size self-similar fractal if $S: K=K$. Let us limit our considerations to fractals in which the self-similarity can be realized only via linear maps, i.e. by transformations which point $\boldsymbol{r}=\left(x_{1}, x_{2}, x_{3}\right) \in K \subset R^{3}$ transform into the point $\boldsymbol{r}^{\prime}=\left(x_{1}^{\prime}, x_{2}^{\prime}, x_{3}^{\prime}\right)$ according to the formula $x_{i}^{\prime}=S_{i_{1}} x_{1}+S_{i_{2}} x_{2}+S_{i_{3}} x_{3}$, where $i=1,2,3$. This transformation is represented by matrix $S$. If we orient coordinate axes along the eigenvectors of matrix $S$ (i.e., $\left.\boldsymbol{r}=\left(x_{1}, x_{2}, x_{3}\right) \rightarrow\left(\xi_{1}, \xi_{2}, \xi_{3}\right)\right)$ the transformation takes the form of $S=S_{1} S_{2} S_{3}$. Each $S_{i}$ represents a multiplier along the coordinate axis $\xi_{i}$. Let us consider more general transformations of the type $S^{m, n, l}=\left(S_{1}\right)^{n} \circ\left(S_{2}\right)^{m} \circ\left(S_{3}\right)^{l}$, where $\left(S_{i}\right)^{n}$ denotes $n$-tuple superposition of transformation $S_{i}$, and define a class of infinite "net fractals" $G_{n f}$, for which the relation $S^{m, n, l}: G_{n f}=G_{n f}$ is valid. It can be proven [6] that, when presented in a logarithmic scale, the family of mappings $S^{(m, n, l)}$ is isomorphic with a $3 \mathrm{D}$ crystal lattice. The isomorphic mapping is given by $S^{(m, n, l)} \rightarrow$ $\left(m a_{1}, n a_{2}, l a_{3}\right)$. The very same refers to the placement of the characteristic building blocks of the "net fractal". This means that after transition to the logarithmic coordinates uniform distribution of spins and of electron density is restored [6].

Let us consider a "net fractal" cluster consisting of $N$ localized magnetic moments. Let us discuss the indirect interactions between magnetic moments provided that some electrons in the fractal systems delocalize. As any indirect magnetic interaction is a collective phenomenon we should prove that typical fractals are large enough to establish indirect coupling between magnetic moments. Fortunately, the answer is easy as we know that in the oxide system electrons delocalized on two magnetic ions suffice to form "double exchange" coupling. Moreover, even in bulk systems the effective range of the RKKY coupling never exceeds a few lattice spacings. Therefore we can conclude that typical real fractals are large enough to allow manifestation of the RKKY-like indirect magnetic coupling.

It is natural to assume that the self-similarity of the fractal is reflected also in the symmetry of exchange interactions. Let us now recall the (log-scale) isomorphism of "net fractals" and some crystal lattices. With these 
assumptions the magnetic "net fractal" is mapped onto a crystalline-like spin lattice. We should remember here that in a fractal the electron mobility is restricted to the directions allowed by the internal geometry. This means that we have mapped the magnetic fractal onto a crystal lattice, in which the spins form a percolation cluster separated from the surrounding by the "red bonds". The percolation of the (log-scale) lattice sets restriction on the electron mobility. To account for existence of "red bonds" we are using the fact that the structure of the equation of particle motion on the percolating network has the form of diffusion equation (or the linearized equation of motion for ferromagnetic spins) [7]. The diffusion on the fractal system as a rule involves the possibility of fractional dynamics [8]. The effective Hamiltonian which involves using fractional order derivatives [8] predicts unconventional (nonparabolic) dispersion relation. This in turn causes the value of spectral dimension $d_{\mathrm{s}}$ that scales the formula for electron density of states $n(\varepsilon)$ :

$$
n(\varepsilon) \mathrm{d} \varepsilon \approx\left(\varepsilon-\varepsilon_{0}\right)^{d_{\mathrm{s}} / 2-1} \mathrm{~d} \varepsilon
$$

to become a fraction [9]. The most interesting fact is that in the case of low-dimensional systems the spectral dimension $d_{\mathrm{s}}$ as a rule differs from the geometrical (topological) dimension. Therefore, when mentioning the dimensionality of the system, we should say which notion of dimension we are speaking about.

For the use of further considerations we should point to the reader that indirect magnetic interactions are transmitted via low-energy excitations of the free electrons (or holes). In view of the above it is evident that the indirect magnetic interactions are governed by the values of the effective spectral dimension [4]. With the use of formula (1) the analytical expression for the RKKY exchange integral in the case of arbitrary spectral dimension $\alpha D$ can be found [4]:

$$
\begin{aligned}
& J(\xi)=J_{0} \xi^{\alpha-2}\left[J_{\alpha / 2-1}(\kappa \xi) Y_{\alpha / 2-1}(\kappa \xi)\right. \\
& \left.\quad+J_{\alpha / 2}(\kappa \xi) Y_{\alpha / 2}(\kappa \xi)\right],
\end{aligned}
$$

where $J_{\alpha}(x)$ and $Y_{\alpha}(x)$ are the Bessel and Neumann functions, respectively.

Therefore, we can conclude that the "net fractal" system pictured in the logarithmic coordinates exhibits the RKKY-reminiscent features [4]. The exchange integrals show conventional sign reversal oscillatory behavior. The leading term in the exchange integrals $J(\xi)$ decays with the interspin separation $\xi$ (measured in the log scale) as $J(\xi) \propto \xi^{-\alpha}$. This means that the envelope of the $J(\xi)$ is governed by the spectral dimension $\alpha$.

Another problem that should be discussed is the possibility of magnetic ordering on a fractal substrate. As we know the topological (geometrical) dimension of the dendritic fractals as the rule is lower than two. Thermodynamics predicts no collective ordering at $T>0$ in a system having dimension $D<2$. On the other hand, experiments show that ferromagnetic ordering exists in some fractal systems. For example the phase separation in the doped $\operatorname{Pr}_{1-x} \mathrm{Ca}_{x} \mathrm{MnO}_{3}$ systems results in the for- mation of the ferromagnetic, charge delocalized regions at the nanometer level with the fractal symmetry [10]. To explain this contradiction, let us discuss the way in which the dimensionality enters into thermodynamical formulae.

To any physical systems various definitions of dimension can be proposed. In a description of collective behavior of many-particle system we shall be interested in geometrical dimension, i.e. dimension of the Euclidean space embedding a particle and/or spectral (dynamical) dimension, which is related to the collective excitations of the system. The spectral dimension $d_{\mathrm{s}}$ is defined via the density of states through the formula (1), where the spectral dimension $d_{\mathrm{s}}$ can take any real (i.e. also fractional) value. Let us prove that in the case of non-translation-invariant structures spectral dimension is the best generalization of the Euclidean dimension of the system when dealing with dynamical or thermodynamical properties. To show it, let us consider how the dimensionality enters the thermodynamical quantities. For an ideal Fermi/Bose gas the grand potential reads [11]

$$
\ln \Xi=\int_{0}^{\infty} n(\varepsilon) \ln \left(1 \pm \mathrm{e}^{-\beta \varepsilon}\right) \mathrm{d} \varepsilon .
$$

From Eq. (3) one can easily see that all the information about the dimensionality of the actual system enters thermodynamical formulae via the density of states $n(\varepsilon)$. Thus, as we can see from the definition of $n(\varepsilon)$ the thermodynamical evolution of any system depends on its spectral dimension. It is well known that low-dimensional systems (e.g. so-called quasi-2-dimensional ones or fractals) can exhibit the spectral dimensions $d_{\mathrm{s}}>3$ [11]. As we can have $d_{\mathrm{s}}>3$ we could expect that at least for some fractals there can exist collective phenomena e.g., ferromagnetism at $T>0$ even if the topological dimension $D$ is smaller than two. Formula (2) refers to the $T=0$ case. At finite temperature, acoustic fractons interact with the electron sea smearing out the spin polarization pattern, consequently the interaction is cut off at a length $\Lambda_{\mathrm{T}}$.

\section{Discussion and summary}

The results obtained so far refer to the "net fractals", which are ideal generalizations of some real fractals. Most real fractals consist of a backbone and side branches (dead ends) attached to it. Thus the real fractals differ from the discussed above "net fractals" as they show the log-periodicity at most only along the backbone. Nevertheless, the dendritic fractal should display discussed mechanism and should be active when interaction along the backbone is considered. We believe that our study through the derivation of analytical results can be of considerable help for experimentalists when rapid and accurate estimates of magnetic exchange integrals are needed. Finally, we hope that our results can be helpful in tailoring properties of spintronic devices.

In conclusion, we believe that our results not only point the way to a new understanding of magnetism of 
fractally structured systems but also give practical indications for engineering of fractally structured electronic devices. Even if the considered model does not fit exactly the most of real fractals it is still useful as it gives a deeper insight into the dynamical evolution of fractal systems.

\section{References}

[1] R. Bouzerar, G. Bouzerar, T. Ziman, Phys. Rev. B 73, 024411 (2006).

[2] P. Mahadevan, A. Zunger, D.D. Sarma, Phys. Rev. Lett. 93, 177201 (2004).

[3] T. Balcerzak, J. Magn. Magn. Mater. 310, 1651 (2007).
[4] Z. Bak, R. Jaroszewicz, W. Gruhn, J. Magn. Magn. Mater. 213, 340 (2000).

[5] Z. Bak, Solid State Commun. 118, 43 (2001).

[6] Z. Bak, Phase Transit. 80, 79 (2007).

[7] T. Nakayama, K. Yakubo, R.L. Orbach, Rev. Mod. Phys. 66, 381 (1994).

[8] N. Laskin, Phys. Rev. E 66, 056108 (2002).

[9] Z. Bak, Phys. Rev. B 68, 064511 (2003).

[10] M. Viret, F. Ott, H. Glaettli, L. Pinsard-Gaudart, A. Revcolevschi, Phys. Rev. Lett. 93, 217402 (2004).

[11] Wu-Sheng Dai, Mi Xie, Phys. Lett. A 311, 340 (2003). 